\title{
EP-140
}

\section{Safe and feasible outcomes of cholecystectomy in extremely elderly patients (octogenarians vs. nonagenarians)}

\author{
Nam Kyu $\mathrm{CHOI}^{*}$
}

Department of Hepatobililiary Pancreas Surgery, Chosun University Hospital, Gwangju, Korea

Introduction: Acute cholecystitis is a major complication of biliary lithiasis. Cholecystectomy is the gold standard treatment for gallbladder disease. According to the 2015 Statistics Office in Korea, $4.51 \%$ of the population is aged 80 to 89 years and $0.3 \%$ is aged 90 to 99 years. The safety and feasibility of cholecystectomy in octogenarians have been proven in many studies. In this study, we aimed to assess the outcomes of cholecystectomy in octogenarians and nonagenarians with acute cholecystitis.

Methods: A total of 393 patientsaged 80 to 89 years (352 octogenarians) and 90 to 99 years (41 nonagenarians) diagnosed with acute cholecystitis underwent cholecystectomy between March 2012 and June 2020. All patients were classified according to the Tokyo guidelines. The evaluated parameters included demographic data, surgical outcomes, American Society of Anesthesiologists score (ASA), and Tokyo guidelines. All data were analyzed with SPSS ver. 1.0.0.1406.

Results: All 393 patients were analyzed and divided into two groups according to age: octogenarians (83.57 \pm 2.64 years) and nonagenarians (92.98 \pm 3.15 years). The survival rate was $97.7 \%$ for octogenarians and $97.6 \%$ for nonagenarians. Laparoscopic surgery was performed more in both groups (96.8\% in octogenarians and $92.7 \%$ in nonagenarians) than open surgery (3.2\% in octogenarians and $7.3 \%$ in nonagenarians). The incidences of postoperative complications in the octogenarian and nonagenarian groups were as follows: pneumonia, $5.7 \%$ and $7.3 \%$; bleeding, $1.7 \%$ and $2.4 \%$; gastrointestinal symptoms, $6.0 \%$ and $2.4 \%$;

Conclusions: Cholecystectomy is a safe and efficient procedure for the treatment of acute cholecystitis in both octogenarians and nonagenarians. 